\title{
Application of Functionals in Creating Inequalities
}

\author{
Zlatko Pavić, ${ }^{1}$ Shanhe $\mathrm{Wu}^{2}$ and Vedran Novoselac ${ }^{1}$ \\ ${ }^{1}$ Mechanical Engineering Faculty in Slavonski Brod, University of Osijek, Trg Ivane Brlić Mažuranić 2, 35000 Slavonski Brod, Croatia \\ ${ }^{2}$ Department of Mathematics, Longyan University, Longyan, Fujian 364012, China
}

Correspondence should be addressed to Shanhe Wu; shanhewu@gmail.com

Received 14 July 2016; Accepted 27 September 2016

Academic Editor: Calogero Vetro

Copyright (C) 2016 Zlatko Pavić et al. This is an open access article distributed under the Creative Commons Attribution License, which permits unrestricted use, distribution, and reproduction in any medium, provided the original work is properly cited.

\begin{abstract}
The paper deals with the fundamental inequalities for convex functions in the bounded closed interval. The main inequality includes convex functions and positive linear functionals extending and refining the functional form of Jensen's inequality. This inequality implies the Jensen, Fejér, and, thus, Hermite-Hadamard inequality, as well as their refinements.
\end{abstract}

\section{Introduction}

In our research, we apply the theory of positive linear functionals to convex analysis. Let us remember the initial notions related to positive linear functionals on the space of real functions.

Let $S$ be a nonempty set, and let $\mathbb{F}=\mathbb{F}(S)$ be a subspace of the linear space of all real functions on domain $S$. We assume that space $\mathbb{F}$ contains unit function $u$ defined by $u(s)=1$ for every $s \in S$. Such space $\mathbb{F}$ contains every real constant $\kappa$ within the meaning of $\kappa=\kappa u$ and every composite function $f(g)$ of a function $g \in \mathbb{F}$ and an affine function $f: \mathbb{R} \rightarrow \mathbb{R}$. Actually, if $f(x)=\kappa_{1} x+\kappa_{2}$, then the composition

$$
f(g)=\kappa_{1} g+\kappa_{2} u
$$

belongs to $\mathbb{F}$.

Let $\mathbb{L}=\mathbb{L}(\mathbb{F})$ be the space of all linear functionals on space $\mathbb{F}$. Functional $L \in \mathbb{L}$ is said to be unital (normalized) if $L(u)=1$. Such functional has property $L(\kappa u)=\kappa$ for every real constant $\kappa$. If $g \in \mathbb{F}$ is a function and if $L \in \mathbb{L}$ is a unital functional, then affine function $f: \mathbb{R} \rightarrow \mathbb{R}$ satisfies equality

$$
f(L(g))=L(f(g)) \text {. }
$$

Functional $L \in \mathbb{L}$ is said to be positive (nonnegative) if inequality $L(g) \geq 0$ holds for every nonnegative function $g \in \mathbb{F}$. If a pair of functions $g_{1}, g_{2} \in \mathbb{F}$ satisfies inequality $g_{1}(s) \leq g_{2}(s)$ for every $s \in S$, then it follows that

$$
L\left(g_{1}\right) \leq L\left(g_{2}\right) \text {. }
$$

If $g \in \mathbb{F}$ is a function with the image in interval $[a, b]$ (i.e., $a u \leq g \leq b u)$, then every positive unital functional $L \in \mathbb{L}$ meets inclusion $L(g) \in[a, b]$ (i.e., $a \leq L(g) \leq b$ ). The same is true for each closed interval $I \subseteq \mathbb{R}$.

Introducing a continuous convex function, we can expose the functional form of Jensen's inequality.

Theorem A. Let $g \in \mathbb{F}$ be a function with the image in closed interval $I \subseteq \mathbb{R}$, and let $L \in \mathbb{L}$ be a positive unital functional.

Then each continuous convex function $f: I \rightarrow \mathbb{R}$ such that $f(g) \in \mathbb{F}$ satisfies inequality

$$
f(L(g)) \leq L(f(g))
$$

We will consider convex functions in bounded closed interval $[a, b]$ with endpoints $a<b$. Each point $x \in[a, b]$ can be represented by the unique binomial convex combination

$$
x=\alpha_{x} a+\beta_{x} b
$$

where

$$
\begin{aligned}
& \alpha_{x}=\frac{b-x}{b-a}, \\
& \beta_{x}=\frac{x-a}{b-a} .
\end{aligned}
$$


Convex function $f:[a, b] \rightarrow \mathbb{R}$ is bounded by two lines. The secant line of function $f$ passes through graph points $A(a, f(a))$ and $B(b, f(b))$, and its equation is

$$
f_{a b}^{\mathrm{sec}}(x)=\frac{b-x}{b-a} f(a)+\frac{x-a}{b-a} f(b) .
$$

Let $c \in(a, b)$ be an interior point. The support lines of function $f$ pass through graph point $C(c, f(c))$. Each support line is specified by slope coefficient $\kappa \in\left[f^{\prime}(c-), f^{\prime}(c+)\right]$, and its equation is

$$
f_{c}^{\sup }(x)=\kappa(x-c)+f(c) .
$$

The support-secant line inequality

$$
f_{c}^{\text {sup }}(x) \leq f(x) \leq f_{a b}^{\mathrm{sec}}(x)
$$

holds for every $x \in[a, b]$.

In 1931, Jessen (see $[1,2]$ ) stated the functional form of Jensen's inequality for convex functions in interval $I \subseteq \mathbb{R}$. In 1988, I. Rasa and I. Raşa (see [3]) pointed out that $I$ must be closed otherwise it could happen that $L(g) \notin I$ and that $f$ must be continuous otherwise it could happen that the inequality in formula (4) does not apply. In Theorem A, we have taken into account I. Rasa and I. Raşa’s remarks. Some generalizations of the functional form of Jensen's inequality can be found in [4].

A concise book on functional analysis, which contains an essential overview of operator theory and indicates the importance of positive linear functionals, is certainly the book in [5].

\section{Main Results}

We firstly present the extension of the inequality in formula (4) concerning interval $[a, b]$.

Lemma 1. Let $g \in \mathbb{F}$ be a function with the image in $[a, b]$, and let $L \in \mathbb{L}$ be a positive unital functional.

Then each continuous convex function $f:[a, b] \rightarrow \mathbb{R}$ such that $f(g) \in \mathbb{F}$ satisfies double inequality

$$
f(L(g)) \leq L(f(g)) \leq f_{a b}^{s e c}(L(g)) .
$$

Proof. The point $l=L(g)$ is in interval $[a, b]$. We sketch the proof in two steps depending on the position of $l$.

If $l \in(a, b)$, we take support line $f_{l}^{\text {sup }}$ of $f$ at $l$. By applying positive functional $L$ to the support-secant inequality in formula (9) with $x=g(s)$, where $s \in S$, we get

$$
L\left(f_{l}^{\mathrm{sup}}(g)\right) \leq L(f(g)) \leq L\left(f_{a b}^{\mathrm{sec}}(g)\right) .
$$

By utilizing the affinity of functions $f_{l}^{\text {sup }}$ and $f_{a b}^{\text {sec }}$ via formula (2), the above inequality takes the form

$$
f_{l}^{\text {sup }}(L(g)) \leq L(f(g)) \leq f_{a b}^{\mathrm{sec}}(L(g)),
$$

where the first term

$$
f_{l}^{\text {sup }}(L(g))=f(L(g)) .
$$

If $l \in\{a, b\}$, we rely on the continuity of $f$ using a support line at a point of open interval $(a, b)$ that is close enough to $l$. Given $\varepsilon>0$, we can find $c \in(a, b)$ so that

$$
f(l)-\varepsilon<f_{c}^{\text {sup }}(l) .
$$

By combining the above inequality and the inequality in formula (12) with the support line at $c$, we obtain

$$
f(l)-\varepsilon<f_{c}^{\text {sup }}(l) \leq L(f(g)) \leq f_{a b}^{\text {sec }}(l)=f(l) .
$$

Letting $\varepsilon$ approach zero, we reach the conclusion $L(f(g))=$ $f(l)$. In this case, trivial inequality $f(l) \leq f(l) \leq f(l)$ represents formula (10).

Formula (10) can be expressed in the form which includes the convex combination of interval endpoints $a$ and $b$. The respective form of Lemma 1 is as follows.

Corollary 2. Let $g \in \mathbb{F}$ be a function with the image in $[a, b]$, and let $L \in \mathbb{L}$ be a positive unital functional. Let

$$
l=L(g)=\alpha_{l} a+\beta_{l} b .
$$

Then each continuous convex function $f:[a, b] \rightarrow \mathbb{R}$ such that $f(g) \in \mathbb{F}$ satisfies double inequality

$$
f\left(\alpha_{l} a+\beta_{l} b\right) \leq L(f(g)) \leq \alpha_{l} f(a)+\beta_{l} f(b) .
$$

Proof. As regards to the last terms of formulae (10) and (17), we have

$$
\begin{aligned}
f_{a b}^{\mathrm{sec}}(L(g)) & =\alpha_{l} f_{a b}^{\mathrm{sec}}(a)+\beta_{l} f_{a b}^{\mathrm{sec}}(b) \\
& =\alpha_{l} f(a)+\beta_{l} f(b)
\end{aligned}
$$

because of the affinity of $f_{a b}^{\mathrm{sec}}$ and its coincidence with $f$ at endpoints.

In order to refine the inequality in formula (10), we will combine the secant lines of convex function $f$ with positive unital functionals.

Lemma 3. Let $c \in(a, b)$ be a point.

Then each convex function $f:[a, b] \rightarrow \mathbb{R}$ satisfies the secant lines inequality

$$
\begin{gathered}
\min \left\{f_{a c}^{s e c}(x), f_{c b}^{s e c}(x)\right\} \leq f(x) \\
\leq \max \left\{f_{a c}^{s e c}(x), f_{c b}^{s e c}(x)\right\}
\end{gathered}
$$

for every $x \in[a, b]$

Proof. Cases $x \in[a, c]$ and $x \in[c, b]$ should be considered.

Suppose that function $g \in \mathbb{F}$ has the image in $[a, b]$ and is not identically equal to $a$ or $b$. Such function satisfies inequality $g\left(s_{1}\right) \leq c \leq g\left(s_{2}\right)$ for some number $c \in(a, b)$ and some pair of points $s_{1}, s_{2} \in S$. In that case, we can find a pair of functionals $L_{1}, L_{2} \in \mathbb{L}$ meeting related inequality

$$
L_{1}(g) \leq c \leq L_{2}(g) .
$$


For example, we can take the point evaluations at $s_{1}$ and $s_{2}$, that is, the functionals defined by $L_{1}(q)=q\left(s_{1}\right)$ and $L_{2}(q)=$ $q\left(s_{2}\right)$ for every function $q \in \mathbb{F}$.

In the main theorem, we use functionals $L_{1}$ and $L_{2}$ satisfying the inequality in formula (20).

Theorem 4. Let $c \in(a, b)$ be a point. Let $g \in \mathbb{F}$ be a function with the image in $[a, b]$, and let $L_{1}, L_{2} \in \mathbb{L}$ be positive unital functionals such that $L_{1}(g) \in[a, c]$ and $L_{2}(g) \in[c, b]$. Let $L=\lambda_{1} L_{1}+\lambda_{2} L_{2}$ be a convex combination of $L_{1}$ and $L_{2}$.

Then each continuous convex function $f:[a, b] \rightarrow \mathbb{R}$ such that $f(g) \in \mathbb{F}$ satisfies the series of inequalities

$$
\begin{aligned}
f(L(g)) & \leq \lambda_{1} f\left(L_{1}(g)\right)+\lambda_{2} f\left(L_{2}(g)\right) \leq L(f(g)) \\
& \leq \lambda_{1} f_{a c}^{s e c}\left(L_{1}(g)\right)+\lambda_{2} f_{c b}^{s e c}\left(L_{2}(g)\right) \\
& \leq f_{a b}^{s e c}(L(g)) .
\end{aligned}
$$

Proof. By applying the convexity of $f$ to convex combination $L(g)=\lambda_{1} L_{1}(g)+\lambda_{2} L_{2}(g)$, we get

$$
f(L(g)) \leq \lambda_{1} f\left(L_{1}(g)\right)+\lambda_{2} f\left(L_{2}(g)\right) .
$$

By applying the left-hand side of formula (10) to $L_{1}$ and $L_{2}$, we obtain

$$
\begin{aligned}
& \lambda_{1} f\left(L_{1}(g)\right)+\lambda_{2} f\left(L_{2}(g)\right) \\
& \quad \leq \lambda_{1} L_{1}(f(g))+\lambda_{2} L_{2}(f(g))=L(f(g)) .
\end{aligned}
$$

As the right-hand side of formula (19) with $x=g(s)$, the inequality

$$
f(g(s)) \leq \max \left\{f_{a c}^{\mathrm{sec}}(g(s)), f_{c b}^{\mathrm{sec}}(g(s))\right\}
$$

holds for every $s \in S$. By acting with $L_{1}$ in the above inequality and using assumption $L_{1}(g) \in[a, c]$, we find

$$
L_{1}(f(g)) \leq L_{1}\left(f_{a c}^{\mathrm{sec}}(g)\right)=f_{a c}^{\mathrm{sec}}\left(L_{1}(g)\right),
$$

and similarly, by acting with $L_{2}$ and using the assumption $L_{2}(g) \in[c, b]$, we find

$$
L_{2}(f(g)) \leq L_{2}\left(f_{c b}^{\mathrm{sec}}(g)\right)=f_{c b}^{\mathrm{sec}}\left(L_{2}(g)\right) .
$$

Multiplication by $\lambda_{1}$ and $\lambda_{2}$ and then summation yield

$$
\begin{aligned}
& \lambda_{1} L_{1}(f(g))+\lambda_{2} L_{2}(f(g)) \\
& \quad \leq \lambda_{1} f_{a c}^{\mathrm{sec}}\left(L_{1}(g)\right)+\lambda_{2} f_{c b}^{\mathrm{sec}}\left(L_{2}(g)\right) .
\end{aligned}
$$

Using main secant $f_{a b}^{\text {sec }}$, we reach conclusion

$$
\begin{aligned}
& \lambda_{1} f_{a c}^{\mathrm{sec}}\left(L_{1}(g)\right)+\lambda_{2} f_{c b}^{\mathrm{sec}}\left(L_{2}(g)\right) \\
& \quad \leq \lambda_{1} f_{a b}^{\mathrm{sec}}\left(L_{1}(g)\right)+\lambda_{2} f_{a b}^{\mathrm{sec}}\left(L_{2}(g)\right) \\
& \quad=f_{a b}^{\mathrm{sec}}(L(g)) .
\end{aligned}
$$

Putting together the inequalities in formulae (22), (23), (27), and (28) into a series, we achieve the inequality in formula (21).

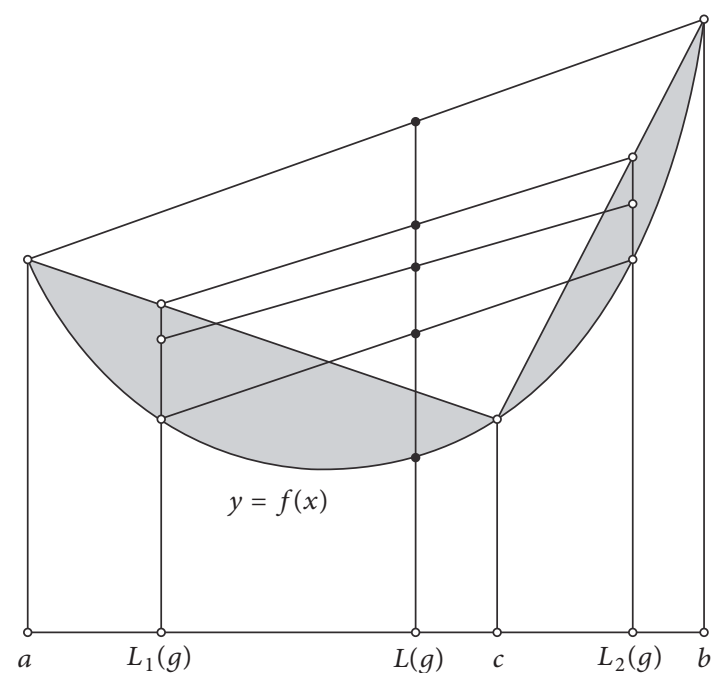

FIGURE 1: Geometric presentation of the inequality in formula (21).

The geometric presentation of the series of inequalities in formula (21) is created in Figure 1. The inequality terms are represented by five black dots above point $L(g)$.

To emphasize interval endpoints $a$ and $b$, we present the following version of Theorem 4 .

Corollary 5. Let $c \in(a, b)$ be a point. Let $g \in \mathbb{F}$ be a function with the image in $[a, b]$, and let $L_{1}, L_{2} \in \mathbb{L}$ be positive unital functionals such that $L_{1}(g) \in[a, c]$ and $L_{2}(g) \in[c, b]$. Let $L=\lambda_{1} L_{1}+\lambda_{2} L_{2}$ be a convex combination of $L_{1}$ and $L_{2}$, and let $l=L(g)=\alpha_{l} a+\beta_{l} b$.

Then each continuous convex function $f:[a, b] \rightarrow \mathbb{R}$ such that $f(g) \in \mathbb{F}$ satisfies the series of inequalities

$$
\begin{aligned}
f\left(\alpha_{l} a+\beta_{l} b\right) & \leq \lambda_{1} f\left(L_{1}(g)\right)+\lambda_{2} f\left(L_{2}(g)\right) \\
& \leq L(f(g)) \leq \alpha f(a)+\beta f(b)+\gamma f(c) \\
& \leq \alpha_{l} f(a)+\beta_{l} f(b),
\end{aligned}
$$

where

$$
\begin{aligned}
& \alpha=\lambda_{1} \frac{c-L_{1}(g)}{c-a}, \\
& \beta=\lambda_{2} \frac{L_{2}(g)-c}{b-c}, \\
& \gamma=\lambda_{1} \frac{L_{1}(g)-a}{c-a}+\lambda_{2} \frac{b-L_{2}(g)}{b-c} .
\end{aligned}
$$

Proof. To calculate coefficients $\alpha, \beta$, and $\gamma$, we include convex combinations $L_{1}(g)=\alpha_{1} a+\gamma_{1} c$ and $L_{2}(g)=\gamma_{2} c+\beta_{2} b$. Then the fourth term of formula (21) takes the form

$$
\begin{aligned}
& \lambda_{1} f_{a c}^{\mathrm{sec}}\left(L_{1}(g)\right)+\lambda_{2} f_{c b}^{\mathrm{sec}}\left(L_{2}(g)\right) \\
& \quad=\lambda_{1} \alpha_{1} f(a)+\lambda_{2} \beta_{2} f(b)+\left(\lambda_{1} \gamma_{1}+\lambda_{2} \gamma_{2}\right) f(c) .
\end{aligned}
$$


Taking the coefficient of $f(a)$ and using formula (6), we calculate

$$
\alpha=\lambda_{1} \alpha_{1}=\lambda_{1} \frac{c-L_{1}(g)}{c-a} .
$$

Similarly we determine $\beta$ and $\gamma$.

Let us finish the section by presenting the generalization of Theorem 4 that uses several secant lines.

Corollary 6. Let $a=c_{0}<c_{1}<\cdots<c_{n-1}<c_{n}=b$ be points. Let $g \in \mathbb{F}$ be a function with the image in $[a, b]$, and let $L_{i} \in \mathbb{L}$ be positive unital functionals such that $L_{i}(g) \in\left[c_{i-1}, c_{i}\right]$ for $i=1, \ldots, n$. Let $L=\sum_{i=1}^{n} \lambda_{i} L_{i}$ be a convex combination of functionals $L_{i}$.

Then each continuous convex function $f:[a, b] \rightarrow \mathbb{R}$ such that $f(g) \in \mathbb{F}$ satisfies the series of inequalities

$$
\begin{aligned}
f(L(g)) & \leq \sum_{i=1}^{n} \lambda_{i} f\left(L_{i}(g)\right) \leq L(f(g)) \\
& \leq \sum_{i=1}^{n} \lambda_{i} f_{c_{i-1} c_{i}}^{s e c}\left(L_{i}(g)\right) \leq f_{a b}^{s e c}(L(g)) .
\end{aligned}
$$

\section{Applications to Integral and Discrete Inequalities}

We firstly utilize Lemma 1 to obtain a very general integral inequality.

Corollary 7. Let $g:[a, b] \rightarrow \mathbb{R}$ be an integrable function with the image in $[a, b]$, and let $h:[a, b] \rightarrow \mathbb{R}$ be a positive integrable function.

Then each convex function $f:[a, b] \rightarrow \mathbb{R}$ satisfies double inequality

$$
\begin{aligned}
f\left(\frac{\int_{a}^{b} g h d x}{\int_{a}^{b} h d x}\right) \leq & \frac{\int_{a}^{b} f(g) h d x}{\int_{a}^{b} h d x} \\
\leq & \frac{\int_{a}^{b}(b-g) h d x}{\int_{a}^{b}(b-a) h d x} f(a) \\
& +\frac{\int_{a}^{b}(g-a) h d x}{\int_{a}^{b}(b-a) h d x} f(b) .
\end{aligned}
$$

Proof. Let $\mathbb{F}$ be the space of all integrable functions over domain $S=[a, b]$. Composition $f(g)$ is bounded in $[a, b]$ and continuous almost everywhere in $[a, b]$. Therefore $f(g)$ is integrable over $[a, b]$, that is, $f(g) \in \mathbb{F}$.

The integrating linear functional $L$ defined by

$$
L(q)=L(q ; h)=\frac{\int_{a}^{b} q h d x}{\int_{a}^{b} h d x}
$$

for every $q \in \mathbb{F}$ is positive and unital. The first term of formula (34) is equal to $f(L(g))$, the second term is equal to $L(f(g))$, and the third term is equal to $f_{a b}^{\mathrm{sec}}(L(g))$. Thus, formula (34) fits into the frame of formula (10), and it is valid for a continuous convex function $f$.

Let us verify that the inequality in formula (34) applies to a convex function which is not continuous at endpoints. We observe the position of point

$$
l=L(g)=\frac{\int_{a}^{b} g h d x}{\int_{a}^{b} h d x} .
$$

If $l \in(a, b)$, then we may utilize continuous extension $\tilde{f}$ of $f /(a, b)$ to $[a, b]$ in formula (34). The first two terms are the same as we use $f$, and the last terms satisfy inequality

$$
\begin{aligned}
\alpha_{l} \tilde{f}(a)+\beta_{l} \tilde{f}(b) & =\tilde{f}_{a b}^{\mathrm{sec}}(l)<f_{a b}^{\mathrm{sec}}(l) \\
& =\alpha_{l} f(a)+\beta_{l} f(b) .
\end{aligned}
$$

So, formula (34) applies to $f$ in this case.

If $l \in\{a, b\}$, then either $g(x)-l \geq 0$ or $g(x)-l \leq 0$ for every $x \in[a, b]$. We rearrange formula (36) to be integral equation

$$
\int_{a}^{b}(g-l) h d x=0
$$

from which it follows that $g(x)=l$ for almost every $x \in[a, b]$. Thus we have that $f(g(x))=f(l)$ for almost every $x \in[a, b]$, and inequality $f(l) \leq f(l) \leq f(l)$ represents formula (34).

Respecting all considerations, we may conclude that the inequality in formula (34) applies to any convex function $f$.

The inequality in formula (34) is the extended version of Jensen's inequality for the ratio of integrals in interval $[a, b]$, as well as the generalized form of the Fejér and HermiteHadamard inequality.

Let us demonstrate the simplifications of the inequality in formula (34) relating to the identity, unit, and symmetric function.

Using $h(x)=1$, we get the extension of the classical integral form of Jensen's inequality (see [6])

$$
\begin{aligned}
f\left(\frac{\int_{a}^{b} g d x}{b-a}\right) \leq & \frac{\int_{a}^{b} f(g) d x}{b-a} \\
\leq & \frac{\int_{a}^{b}(b-g) d x}{(b-a)^{2}} f(a) \\
& +\frac{\int_{a}^{b}(g-a) d x}{(b-a)^{2}} f(b) .
\end{aligned}
$$

Using identity function $g(x)=x$ and function $h(x)$, satisfying equation $h(x)=h(a+b-x)$, which represents the symmetry with a center at midpoint $(a+b) / 2$, we have the classical form of Fejér inequality (see [7])

$$
f\left(\frac{a+b}{2}\right) \leq \frac{\int_{a}^{b} f h d x}{\int_{a}^{b} h d x} \leq \frac{f(a)+f(b)}{2} .
$$


Namely, as a consequence of the symmetry we have

$$
\begin{aligned}
\frac{\int_{a}^{b} x h d x}{\int_{a}^{b} h d x}= & \frac{\int_{a}^{b}(x-(a+b) / 2) h d x}{\int_{a}^{b} h d x} \\
& +\frac{\int_{a}^{b}((a+b) / 2) h d x}{\int_{a}^{b} h d x}=\frac{a+b}{2}
\end{aligned}
$$

because

$$
\int_{a}^{b}\left(x-\frac{a+b}{2}\right) h d x=0
$$

Following formula (41), we can conclude that the Fejér inequality is valid for function $h$ satisfying weaker condition $\int_{a}^{b} x h d x / \int_{a}^{b} h d x=(a+b) / 2$.

Using $h(x)=1$ in Fejér's inequality in formula (40), we obtain the classical form of Hermite-Hadamard inequality $($ see $[8,9])$

$$
f\left(\frac{a+b}{2}\right) \leq \frac{\int_{a}^{b} f d x}{b-a} \leq \frac{f(a)+f(b)}{2}
$$

To obtain refinements of the inequality in formula (34), we use point $c \in(a, b)$ and apply Theorem 4 .

Corollary 8. Let $c \in(a, b)$ be a point. Let $g:[a, b] \rightarrow \mathbb{R}$ be an integrable function such that $g(x) \in[a, c]$ for $x \in[a, c]$ and $g(x) \in[c, b]$ for $x \in[c, b]$, and let $h:[a, b] \rightarrow \mathbb{R}$ be a positive integrable function.

Then each convex function $f:[a, b] \rightarrow \mathbb{R}$ satisfies the series of inequalities

$$
\begin{aligned}
& f\left(\frac{\int_{a}^{b} g h d x}{\int_{a}^{b} h d x}\right) \\
& \leq \frac{\int_{a}^{c} h d x}{\int_{a}^{b} h d x} f\left(\frac{\int_{a}^{c} g h d x}{\int_{a}^{c} h d x}\right) \\
& \quad+\frac{\int_{c}^{b} h d x}{\int_{a}^{b} h d x} f\left(\frac{\int_{c}^{b} g h d x}{\int_{c}^{b} h d x}\right) \leq \frac{\int_{a}^{b} f(g) h d x}{\int_{a}^{b} h d x} \\
& \leq \frac{\int_{a}^{c}(c-g) h d x}{\int_{a}^{b}(c-a) h d x} f(a)+\frac{\int_{c}^{b}(g-c) h d x}{\int_{a}^{b}(b-c) h d x} f(b) \\
& \quad+\left(\frac{\int_{a}^{c}(g-a) h d x}{\int_{a}^{b}(c-a) h d x}+\frac{\int_{c}^{b}(b-g) h d x}{\int_{a}^{b}(b-c) h d x}\right) f(c) \\
& \leq \frac{\int_{a}^{b}(b-g) h d x}{\int_{a}^{b}(b-a) h d x} f(a)+\frac{\int_{a}^{b}(g-a) h d x}{\int_{a}^{b}(b-a) h d x} f(b) .
\end{aligned}
$$

Proof. Just as in Corollary 7 , we use $\mathbb{F}$ as the space of all integrable functions in interval $[a, b]$. In order to apply Theorem 4, we define integrating linear functionals

$$
\begin{aligned}
& L_{1}(q)=L_{1}(q ; h)=\frac{\int_{a}^{c} q h d x}{\int_{a}^{c} h d x}, \\
& L_{2}(q)=L_{2}(q ; h)=\frac{\int_{c}^{b} q h d x}{\int_{c}^{b} h d x}
\end{aligned}
$$

for every $q \in \mathbb{F}$. Functionals $L_{1}$ and $L_{2}$ are positive and unital.

Since $g(x) \in[a, c]$ for $x \in[a, c]$, point $L_{1}(g)$ falls into $[a, c]$, and similarly point $L_{2}(g)$ falls into $[c, b]$. Using coefficients

$$
\begin{aligned}
& \lambda_{1}=\frac{\int_{a}^{c} h d x}{\int_{a}^{b} h d x}, \\
& \lambda_{2}=\frac{\int_{c}^{b} h d x}{\int_{a}^{b} h d x}
\end{aligned}
$$

and functional convex combination $L=\lambda_{1} L_{1}+\lambda_{2} L_{2}$, we get

$$
\begin{aligned}
L(g) & =\lambda_{1} L_{1}(g)+\lambda_{2} L_{2}(g)=\frac{\int_{a}^{b} g h d x}{\int_{a}^{b} h d x}, \\
L(f(g)) & =\frac{\int_{a}^{b} f(g) h d x}{\int_{a}^{b} h d x} .
\end{aligned}
$$

By further calculating the functional terms according to formula (21), we obtain the integral terms of formula (44).

The series of inequalities in formula (44) with $g(x)=x$ and $h(x)=1$ gives the refinement of the Hermite-Hadamard inequality in formula (43) as follows:

$$
\begin{aligned}
f\left(\frac{a+b}{2}\right) \leq & \frac{c-a}{b-a} f\left(\frac{a+c}{2}\right)+\frac{b-c}{b-a} f\left(\frac{b+c}{2}\right) \\
\leq & \frac{\int_{a}^{b} f d x}{b-a} \\
\leq & \frac{c-a}{2(b-a)} f(a)+\frac{b-c}{2(b-a)} f(b) \\
& +\frac{1}{2} f(c) \leq \frac{f(a)+f(b)}{2} .
\end{aligned}
$$

The above refinement holds for each $c \in(a, b)$. The version of the above refinement was obtained in [10] by using the Jensen type inequality for convex combinations with the common center. That inequality was used to refine some important means.

The inequality in formula (44) with identity function $g(x)=x$ and a symmetric function satisfying equation 
$h(x)=h(a+b-x)$ provides the refinements of the Fejer inequality in formula (40).

At the end, let us present the discrete version of Corollary 8 . Point evaluations $g\left(x_{i}\right)$ and $h\left(x_{i}\right)$ will be shortened by $g_{i}$ and $h_{i}$, respectively.

Corollary 9. Let $c \in(a, b)$ be a point. Let $g:[a, b] \rightarrow \mathbb{R}$ be a function such that $g(x) \in[a, c]$ for $x \in[a, c]$ and $g(x) \in[c, b]$ for $x \in[c, b]$, and let $h:[a, b] \rightarrow \mathbb{R}$ be a positive function. Let $x_{1}, \ldots, x_{k} \in[a, c]$ and $x_{k+1}, \ldots, x_{n} \in[c, b]$ be points.

Then each convex function $f:[a, b] \rightarrow \mathbb{R}$ satisfies the series of inequalities

$$
\begin{aligned}
f( & \left.\frac{\sum_{i=1}^{n} g_{i} h_{i}}{\sum_{i=1}^{n} h_{i}}\right) \\
\leq & \frac{\sum_{i=1}^{k} h_{i}}{\sum_{i=1}^{n} h_{i}} f\left(\frac{\sum_{i=1}^{k} g_{i} h_{i}}{\sum_{i=1}^{k} h_{i}}\right) \\
& +\frac{\sum_{i=k+1}^{n} h_{i}}{\sum_{i=1}^{n} h_{i}} f\left(\frac{\sum_{i=k+1}^{n} g_{i} h_{i}}{\sum_{i=k+1}^{n} h_{i}}\right) \leq \frac{\sum_{i=1}^{n} f\left(g_{i}\right) h_{i}}{\sum_{i=1}^{n} h_{i}} \\
\leq & \frac{\sum_{i=1}^{k}\left(c-g_{i}\right) h_{i}}{\sum_{i=1}^{n}(c-a) h_{i}} f(a)+\frac{\sum_{i=k+1}^{n}\left(g_{i}-c\right) h_{i}}{\sum_{i=1}^{n}(b-c) h_{i}} f(b) \\
& +\left(\frac{\sum_{i=1}^{k}\left(g_{i}-a\right) h_{i}}{\sum_{i=1}^{n}(c-a) h_{i}}+\frac{\sum_{i=k+1}^{n}\left(b-g_{i}\right) h_{i}}{\sum_{i=1}^{n}(b-c) h_{i}}\right) f(c) \\
\leq & \frac{\sum_{i=1}^{n}\left(b-g_{i}\right) h_{i}}{\sum_{i=1}^{n}(b-a) h_{i}} f(a)+\frac{\sum_{i=1}^{n}\left(g_{i}-a\right) h_{i}}{\sum_{i=1}^{n}(b-a) h_{i}} f(b) .
\end{aligned}
$$

Proof. Let $\mathbb{F}$ be the space of all real functions on domain $S=$ $[a, b]$. We can apply the proof of Corollary 8 to summarizing linear functionals

$$
\begin{aligned}
& L_{1}(q)=L_{1}(q ; h)=\frac{\sum_{i=1}^{k} q_{i} h_{i}}{\sum_{i=1}^{k} h_{i}}, \\
& L_{2}(q)=L_{2}(q ; h)=\frac{\sum_{i=k+1}^{n} q_{i} h_{i}}{\sum_{i=k+1}^{n} h_{i}}
\end{aligned}
$$

acting to every $q \in \mathbb{F}$, and coefficients

$$
\begin{aligned}
& \lambda_{1}=\frac{\sum_{i=1}^{k} h_{i}}{\sum_{i=1}^{n} h_{i}}, \\
& \lambda_{2}=\frac{\sum_{i=k+1}^{n} h_{i}}{\sum_{i=1}^{n} h_{i}} .
\end{aligned}
$$

Functionals $L_{1}$ and $L_{2}$ are certainly positive and unital.

The inequality in formula (49) is the extension and refinement of the famous discrete form of Jensen's inequality (see [11]).

\section{Competing Interests}

The authors declare that they have no competing interests.

\section{Acknowledgments}

The work of the first and third author has been fully supported by Mechanical Engineering Faculty in Slavonski Brod and Croatian Science Foundation under Project HRZZ-5435. The work of the second author has been supported by the Natural Science Foundation of Fujian province of China under Grant no. 2016J01023. The authors would like to thank Velimir Pavić who has graphically prepared Figure 1.

\section{References}

[1] B. Jessen, "Bemærkninger om konvekse Funktioner og Uligheder imellem Middelværdier I," Matematisk Tidsskrift B, pp. 1728, 1931.

[2] B. Jessen, "Bemærkninger om konvekse Funktioner og Uligheder imellem Middelværdier II," Matematisk Tidsskrift B, pp. 8495, 1931.

[3] I. Rasa and I. Raşa, "A note on Jessen's inequality, Itinerant Seminar on Functional Equations, Approximation and Convexity, Universitatea Babes-Bolyai," in I. Raşa, pp. 275-280, Romania, Cluj-Napoca, 1988.

[4] Z. Pavić, "Generalizations of the functional form of Jensen's inequality," Advances in Inequalities and Applications, vol. 2014, article 33, 2014.

[5] W. Arveson, A Short Course on Spectral Theory, Springer, New York, NY, USA, 2002.

[6] J. L. W. V. Jensen, "Sur les fonctions convexes et les inégalités entre les valeurs moyennes," Acta Mathematica, vol. 30, no. 1, pp. 175-193, 1906.

[7] L. Fejér, "Über die fourierreihen II," Mathematischer und Naturwissenschafticher Anzeiger der Ungarischen Akademie der Wissenschaften, vol. 24, pp. 369-390, 1906 (Hungarian).

[8] Ch. Hermite, "Sur deux limites d'une intégrale définie," Mathesis, vol. 3, p. 82, 1883.

[9] J. Hadamard, "Étude sur les propriétés des fonctions enti $\mu$ eres et en particulier d'une function considerée par Riemann," Journal de Mathématiques Pures et Appliquées, vol. 58, pp. 171-215, 1893.

[10] Z. Pavić, "Improvements of the Hermite-HADamard inequality," Journal of Inequalities and Applications, vol. 2015, article 222, 2015.

[11] J. L. W. V. Jensen, “Om konvekse Funktioner og Uligheder mellem Middelværdier," Nyt Tidsskrift for Matematik B, vol. 16, pp. 49-68, 1905. 


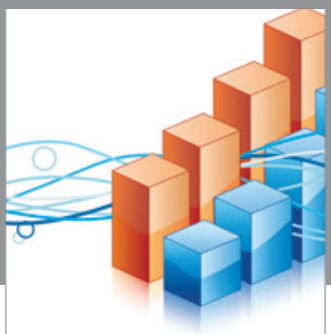

Advances in

Operations Research

vatem alat4

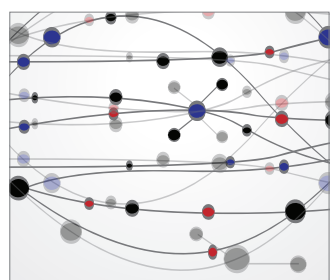

\section{The Scientific} World Journal
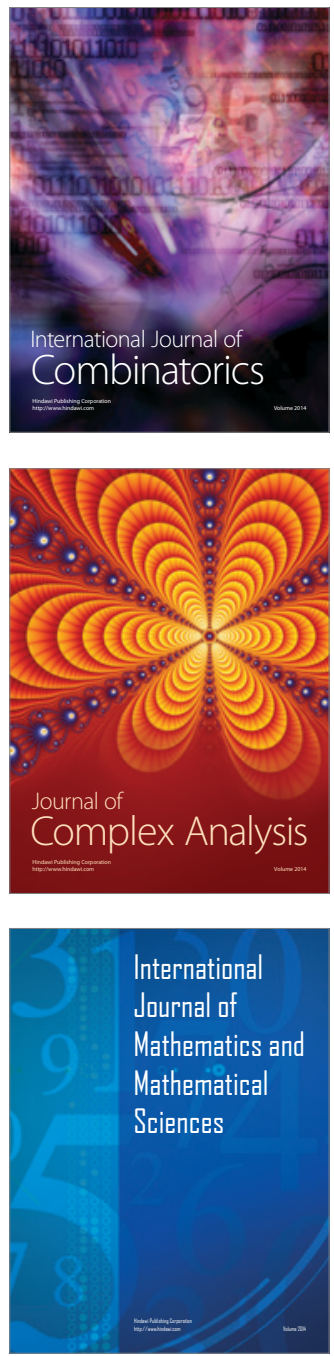
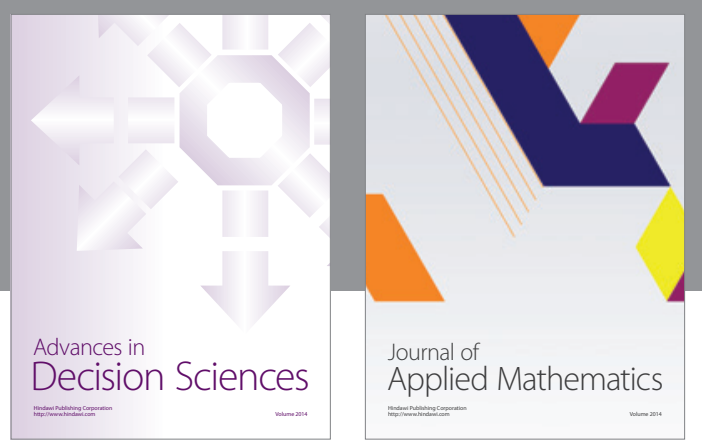

Algebra

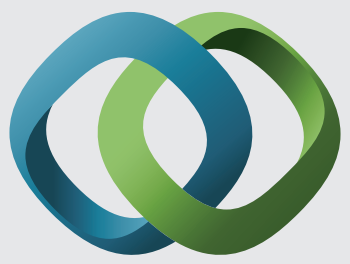

\section{Hindawi}

Submit your manuscripts at

http://www.hindawi.com
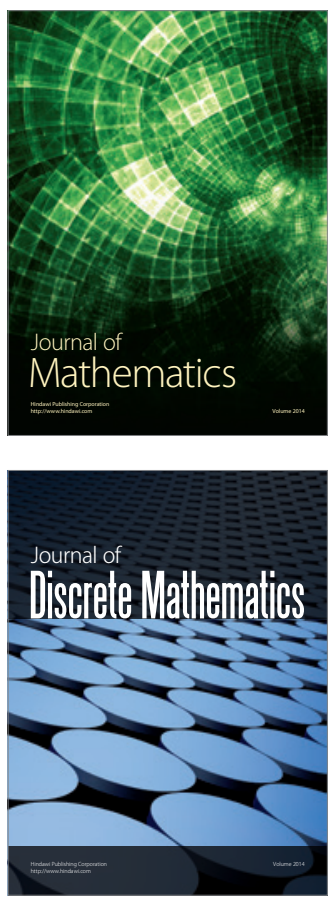

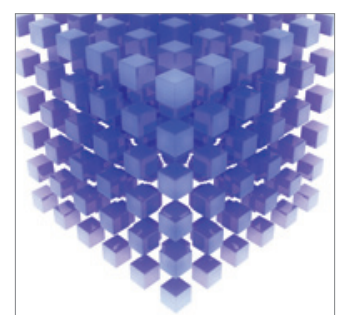

Mathematical Problems in Engineering
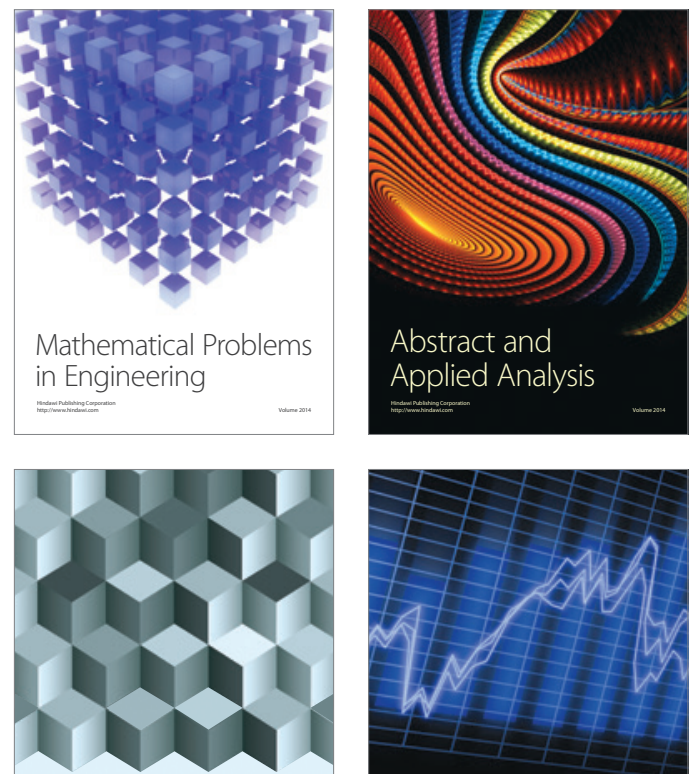

Journal of

Function Spaces

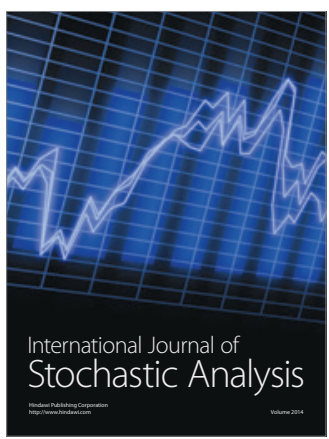

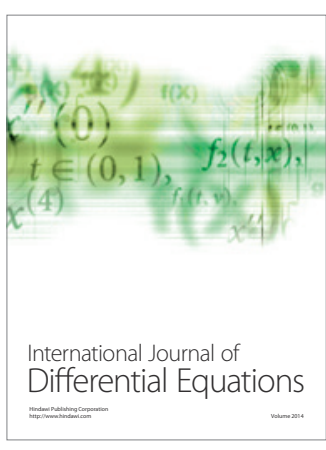
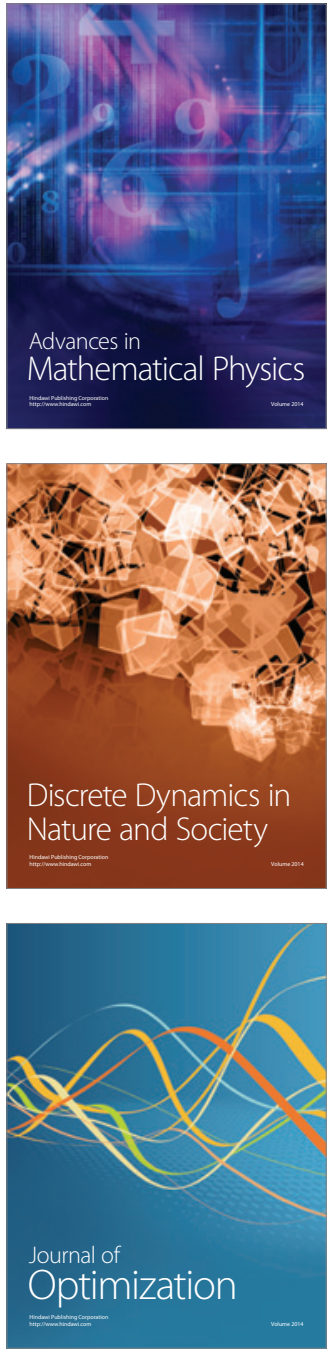